\title{
NOTAS
}

\section{Influencia de la densidad del arbolado sobre el desfronde y su reciclado en pinares de repoblación del norte de España}

\author{
Effects of stand density on litterfall and its turnover in pine plantations in northern Spain
}

\author{
Teresa Bueis ${ }^{\text {a,b }}$, Felipe Bravo ${ }^{\text {a,c }}$, Valentín Pando ${ }^{\text {a,d }}, M^{\mathrm{a}}$ Belén Turrión ${ }^{\mathrm{a}, \mathrm{b}}$ * \\ ${ }^{a}$ Universidad de Valladolid, Instituto Universitario de Investigación en Gestión Forestal Sostenible, Palencia, España.

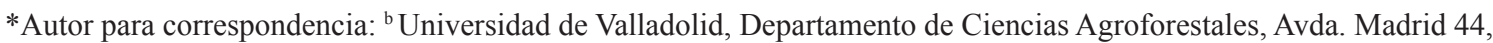 \\ 34004 Palencia, España, tel.: +34979108331, bturrion@agro.uva.es \\ ${ }^{\mathrm{c}}$ Universidad de Valladolid, Departamento de Producción Vegetal y Silvopascicultura. Palencia, España. \\ ${ }^{d}$ Universidad de Valladolid, Departamento de Estadística e Investigación Operativa, Palencia, España.
}

\section{SUMMARY}

The main objective of this work was to study the dynamics of litterfall and litter decomposition in Pinus sylvestris and Pinus halepensis plantations in Castilla y León Region (Spain) as well as the effect of local density on these parameters. Four stands of each species were selected and eight plots were established on each stand. A littertrap and 15 litterbags were set on each plot. Litterfall was collected monthly and a litterbag was removed every three months. The dry weight of litterfall was determined separately for each fraction. The loss of weight over time of needles in the litterbags was also determined. Finally, the relationship between local basal area in the plots and the studied parameters was assessed. The obtained results show significant and positive effects of basal area on litterfall and significant and negative effects on decay rate of needles in the litterbags.

Key words: basal area, litterbag, Pinus halepensis, Pinus sylvestris, littertrap.

\section{RESUMEN}

El objetivo del presente trabajo fue estudiar las dinámicas de desfronde y descomposición de acículas en plantaciones de Pinus halepensis y Pinus sylvestris de Castilla y León (España) así como el efecto de la densidad de las masas sobre dichos parámetros. Para ello se seleccionaron cuatro rodales de cada una de las especies estudiadas y en cada uno de ellos se establecieron ocho parcelas considerando un gradiente de áreas basimétricas, estudiándose un total de 32 parcelas. En el centro de cada parcela se situó una trampa de desfronde y 15 bolsas de descomposición. El desfronde contenido en las trampas se recogió mensualmente. Trimestralmente se retiró una bolsa de descomposición de cada parcela. Se determinó el peso seco de desfronde separado en las distintas fracciones así como la pérdida de peso de las bolsas de descomposición a lo largo del tiempo. Finalmente se estudió la relación existente entre el área basimétrica local de las parcelas y los parámetros antes descritos. Se constató la existencia de diferencias significativas en las cantidades de desfronde y en la tasa de descomposición de las acículas contenidas en las bolsas en función del área basimétrica de la parcela.

Palabras clave: área basimétrica, litterbag, Pinus halepensis, Pinus sylvestris, trampa de desfronde.

\section{INTRODUCCIÓN}

Pinus halepensis Mill. y Pinus sylvestris L. son dos especies ampliamente utilizadas en repoblaciones productoras y protectoras en España. Pinus halepensis es una especie mediterránea, tolerante a la sequía que se ha plantado frecuentemente en zonas áridas o semiáridas para proteger de la erosión y mejorar suelos pobres o degradados. Pinus sylvestris es la especie de pino de mayor distribución mundial y las masas españolas constituyen el límite meridional de su distribución.

El desfronde procedente del arbolado constituye la principal entrada de materia orgánica al suelo. La cantidad de desfronde producido por la masa forestal y el retorno al suelo de los nutrientes que contiene a través de la descomposición son elementos determinantes para la fertilidad del suelo. Es fundamental conocer las tasas de desfronde y descomposición de las masas, así como la relación existente entre el desfronde y su descomposición y la densidad de las masas para asegurar el mantenimiento de la sostenibilidad a través de la gestión forestal. La producción de desfronde disminuye a medida que se reduce el área basimétrica de las masas (Roig et al. 2005, Kunhamu et al. 2009, Navarro et al. 2013), igualmente la descomposición de dicho material se ve influida por el distinto microclima ocasionado 
bajo masas de distintas densidades, aunque el signo de esta relación no siempre es el mismo (Aceñolaza y GallardoLancho 1995, Roig et al. 2005, Kunhamu et al. 2009).

El presente trabajo se plantea a la vista de la escasez de información disponible acerca de las dinámicas de desfronde en las masas de repoblación de $P$. halepensis y $P$. sylvestris de Castilla y León (Gallardo-Lancho et al. 1989, Gallardo-Lancho y Santa-Regina 1991). Dado que la producción de hojarasca es función, entre otros factores, de las condiciones climáticas, es difícil establecer un patrón general en zonas con una variabilidad climática elevada como es la zona Mediterránea (Martínez-Alonso et al. 2007).

Asimismo, se pretende arrojar luz sobre el efecto del área basimétrica de las masas determinado por la gestión forestal de las mismas, sobre las dinámicas de desfronde y descomposición de acículas a la vista de la existencia de resultados contradictorios en la bibliografía (Kunhamu et al. 2009, Lado-Monserrat et al. 2015).

Como primera hipótesis en el presente trabajo se considera que la producción de hojarasca y el momento de desfronde en las masas de estudio diferirán a las observadas para estas mismas especies en otras zonas de España. Como segunda hipótesis se plantea que la espesura de las masas (medida a través del área basimétrica) tendrá un efecto significativo sobre el desfronde así como sobre la descomposición de acículas en las masas de P. halepensis y $P$. sylvestris de los páramos (calizos y ácidos respectivamente) de Castilla y León.

\section{MÉTODOS}

En este trabajo se estudiaron cuatro rodales de $P$. sylvestris ubicados en los páramos ácidos (rañas) y cuatro rodales de $P$. halepensis ubicados en los páramos calizos de la región de Castilla y León en España, todos ellos procedentes de repoblación (figura 1).

Según el índice de Vernet el clima de las zonas de estudio es Mediterráneo y Submediterráneo. Las zonas de estudio de $P$. halepensis presentan una temperatura media anual de $12{ }^{\circ} \mathrm{C}$ y una precipitación anual de $445 \mathrm{~mm}$. Las de $P$. sylvestris presentan una temperatura media anual de $9{ }^{\circ} \mathrm{C}$ y una precipitación anual de $778 \mathrm{~mm}$ (Ninyerola et al. 2005).

En cada uno de los ocho rodales se establecieron ocho parcelas circulares de $6 \mathrm{~m}$ de radio cubriendo un gradiente de áreas basimétricas (cuadro 1). Se midió el diámetro normal (a 1,3 m de altura) de todos los árboles presentes en cada parcela para calcular el área basimétrica local $\left(\mathrm{AB}_{\text {loca }}\right)$. En el centro de cada parcela se estableció una trampa de desfronde y 15 bolsas de descomposición elaboradas con malla de 1,5 mm de luz. Las bolsas de descomposición se rellenaron con $5 \mathrm{~g}$ de acículas senescentes recién caídas y secadas al aire. El experimento sobre $P$. halepensis se estableció en octubre de 2013 y el de $P$. sylvestris en octubre de 2014. En este trabajo se presentan resultados correspondientes al periodo de estudio comprendido entre el establecimiento de los experimentos y octubre de 2015 (dos años para $P$. halepensis y un año para $P$. sylvestris).

Mensualmente se llevó a cabo la recogida del desfronde de las trampas y en el momento de recoger el desfronde se midió la temperatura y la humedad de los $10 \mathrm{~cm}$ superficiales de suelo mediante sondas de temperatura y humedad. Cada tres meses se extrajo una bolsa de descomposición de cada una de las parcelas. Todas las muestras se llevaron al laboratorio y se secaron en estufa a $65^{\circ} \mathrm{C}$ hasta peso constante (Lado-Monserrat et al. 2015). Las muestras de desfronde se separaron en las siguientes fracciones: ací-

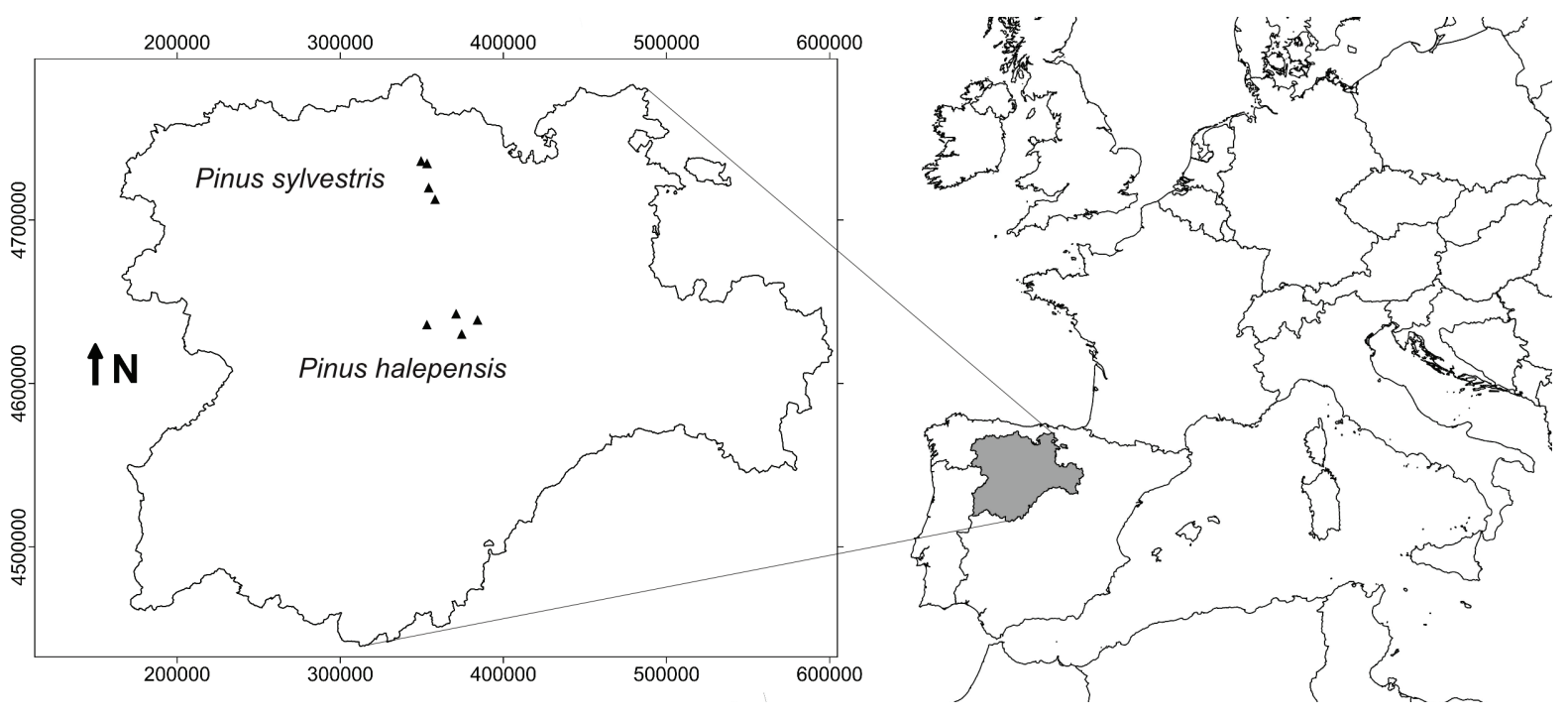

Figura 1. Localización de los rodales de Pinus sylvestris y Pinus halepensis estudiados (coordenadas UTM, huso 30, sistema de referencia ETRS89).

Location of the stands (UTM, ETRS89). 
Cuadro 1. Características de las parcelas estudiadas ${ }^{a}$.

Characteristics of the stands.

\begin{tabular}{llcccc}
\hline Parámetro & \multicolumn{1}{c}{ Especie } & Valor medio & Desviación estándar & Mínimo & Máximo \\
\hline \multirow{2}{*}{ Edad (años) } & Pinus sylvestris & 49,8 & 10,6 & 34 & 57 \\
& Pinus halepensis & 56,5 & 5,4 & 50 & 63 \\
\multirow{2}{*}{ Dap (cm) } & Pinus sylvestris & 25,3 & 3,4 & 21,4 & 36,9 \\
& Pinus halepensis & 24,7 & 3,6 & 18,8 & 32,1 \\
\multirow{2}{*}{$\mathrm{Hm}(\mathrm{m})$} & Pinus sylvestris & 14,0 & 2,7 & 11,4 & 17,7 \\
& Pinus halepensis & 8,4 & 1,8 & 5,9 & 9,7 \\
\multirow{2}{*}{ Cobertura (\%) } & Pinus sylvestris & 85,0 & 16,3 & 25 & 100 \\
& Pinus halepensis & 71,3 & 24,7 & 20 & 100 \\
$\mathrm{AB}_{\text {local }}\left(\mathrm{m}^{2} \mathrm{ha}^{-1}\right)$ & Pinus sylvestris & 29,1 & 13,1 & 6,9 & 50,4 \\
& Pinus halepensis & 33,9 & 17,8 & 5,6 & 77,0 \\
\hline
\end{tabular}

a Dap: diámetro a la altura del pecho; Hm: altura media; Cobertura: fracción de cabida cubierta; $\mathrm{AB}_{\text {local }}$ : área basimétrica local.

culas, corteza, ramillos, piñas, yemas, flores y piñones. Se registró el peso de cada una de las fracciones del desfronde y de las acículas contenidas en las bolsas extraídas. Los datos de pérdida de peso de las bolsas de descomposición a lo largo del tiempo se ajustaron a la ecuación exponencial de Olson (1963) (ecuación 1):

$$
X=X_{0} e^{-k t}
$$

Donde:

$\mathrm{X}=$ Peso de la hojarasca extraída de la bolsa al cabo del tiempo $t$ (meses)

$\mathrm{X}_{0}=$ Peso inicial de la hojarasca introducida en la bolsa $(\mathrm{g})$ $\mathrm{k}=$ Tasa de descomposición de la hojarasca $\left(\mathrm{meses}^{-1}\right)$

Asimismo se estimó el tiempo de vida medio $\left(\mathrm{t}_{0,5}\right)$ de la hojarasca (tiempo necesario para que se produzca una pérdida de peso del $50 \%$ ) a partir de los valores de $\mathrm{k}$ y a través de la ecuación 2 (Olson 1963):

$$
\mathrm{t}_{0,5}=\frac{\ln 0,5}{-\mathrm{k}}
$$

Se estudió la influencia del $\mathrm{AB}_{\text {local }}$ sobre dichos parámetros $\left(\mathrm{k} \mathrm{y}_{0,5}\right)$ para las dos especies por separado.

Para el estudio del desfronde se utilizó un modelo lineal mixto de análisis de la varianza con un factor intersujetos aleatorio (masa) con ocho réplicas (ocho parcelas por masa), un regresor $\left(\mathrm{AB}_{\text {local }}\right)$ y un factor intra-sujetos de medidas repetidas (mes). Se rechazó la hipótesis de normalidad de los datos y, por tanto, se transformaron a través del logaritmo. La formulación matemática del modelo fue la expresada por la ecuación 3 :

$$
Y_{i j ; t}=\mu+\alpha_{i}+\tau_{t}+\beta X_{i j}+\varepsilon_{i j ; t}
$$

Donde:

$i=1,2,3,4$ para las cuatro masas

$j=1,2, \ldots, 8$ para las ocho parcelas dentro de cada masa

$t=1,2, \ldots, 24$ meses en $P$. halepensis y $t=1,2, \ldots, 12$ meses

en $P$. sylvestris

$Y_{i j ; t}=$ Logaritmo del peso de desfronde en la parcela $j$ de la masa $i$ en el mes $t\left(\mathrm{~kg} \mathrm{ha}^{-1}\right)$

$\mu=$ Efecto de media general

$\alpha_{i}=$ Efecto aleatorio de la masa $i$, con $\alpha_{i} \sim N\left(0, \sigma_{S}^{2}\right)$

$\tau_{t}=$ Efecto principal del mes $t$

$X_{i j}=\mathrm{AB}_{\text {local }}$ de la parcela $j$ de la masa $i\left(\mathrm{~m}^{2} \mathrm{ha}^{-1}\right)$

$\beta=$ Tasa lineal de cambio en el logaritmo del peso de desfronde por unidad de $\mathrm{AB}_{\text {local }}$

$\varepsilon_{i j: t}=$ Error aleatorio en el logaritmo del peso de desfronde de la parcela $j$ de la masa $i$ en el mes $t$, con $\varepsilon_{i j ; t} \sim N\left(0, \sigma^{2}\right)$ y con estructura de varianzas auto regresiva de orden $1 \mathrm{AR}(1)$.

Para el estudio de la descomposición a través de los parámetros $\mathrm{k} \mathrm{y} \mathrm{t}_{0,5}$ se utilizó un modelo lineal mixto de análisis de la varianza con un factor inter-sujetos aleatorio (masa) con ocho réplicas (parcelas) y un regresor $\left(\mathrm{AB}_{\text {local }}\right)$. La formulación matemática del modelo fue la expresada por la ecuación 4 :

$$
Y_{i j}=\mu+\alpha_{i}+\beta X_{i j}+\varepsilon_{i j}
$$

Donde:

$i=1,2,3,4$ para las cuatro masas

$j=1,2, \ldots, 8$ para las ocho parcelas dentro de cada masa

$Y_{i j}=$ Valor del parámetro en la parcela $j$ de la masa $i$

$\mu=$ Efecto de media general

$\alpha_{\mathrm{i}}=$ Efecto aleatorio de la masa $i$, con $\alpha_{i} \sim N\left(0, \sigma_{S}^{2}\right)$

$X_{i j}=\mathrm{AB}_{\text {local }}$ de la parcela $j$ de la masa $i\left(\mathrm{~m}^{2} \mathrm{ha}^{-1}\right)$

$\beta=$ Tasa lineal de cambio en el valor del parámetro por unidad de $\mathrm{AB}_{\text {local }}$

$\varepsilon_{i j}=$ Error aleatorio en el valor del parámetro para la parce-

la $j$ de la masa $i$, con $\varepsilon_{i j ; t} \sim N\left(0, \sigma^{2}\right)$ 
Se estudiaron las correlaciones existentes entre el $\mathrm{AB}_{1}$ cal de las parcelas y la temperatura y humedad del suelo a través del coeficiente de correlación de Pearson. El tratamiento estadístico de los datos se realizó con el programa estadístico SAS.

\section{RESULTADOS}

Fenología. El desfronde medio de acículas observado en los dos años de estudio en las parcelas de $P$. halepensis fue de $2.144 \mathrm{~kg} \mathrm{ha}^{-1}$ mientras que en $P$. sylvestris el desfronde medio de acículas durante el año estudiado fue de 2.357 $\mathrm{kg} \mathrm{ha}^{-1}$. En las masas de ambas especies el mes de mayor desfronde fue agosto ( $836 \mathrm{~kg} \mathrm{ha}^{-1}$ promedio en $P$. sylvestris y $635 \mathrm{~kg} \mathrm{ha}^{-1}$ en $P$. halepensis). El pico de mayor desfronde se extendió a los meses de julio a septiembre en $P$. sylvestris y de junio a agosto en $P$. halepensis (figura 2).

Relación desfronde-descomposición con variables dasométricas. El modelo lineal mixto de análisis de la varianza mostró que el efecto del $\mathrm{AB}_{\text {local }}$ sobre el desfronde de ambas especies es significativo $\left(P<0,0001 ; \beta_{(P . \text { halepensis })}=\right.$ 0,0339 y $\left.\beta_{(P . \text { sylvestris })}=0,0197\right)$ de manera que a mayor $\mathrm{AB}_{\text {lo }}$ cal, mayor es la tasa de desfronde. Esta tendencia se puede observar también en la figura 3. Asimismo, el efecto del $\mathrm{AB}_{\text {local }}$ sobre los parámetros relacionados con la descomposición de las acículas $\left(\mathrm{k} \mathrm{y} \mathrm{t}_{0,5}\right)$ en $P$. halepensis resultó significativo $(P<0,0001)$ de forma que a mayor $\mathrm{AB}_{\text {local }}$ menor es la tasa de descomposición y mayor el tiempo de vida medio de la hojarasca (figura 4). No se encontró un efecto significativo del $\mathrm{AB}_{\text {local }}$ sobre ninguno de los parámetros relativos a la descomposición de las acículas en $P$. sylvestris (cuadro 2, figura 4).

Se hallaron correlaciones significativas y negativas entre los valores de $\mathrm{AB}_{\text {local }} \mathrm{y}$ de humedad del suelo tanto para las parcelas de $P$. sylvestris $(\mathrm{r}=-0,209 ; P=0,0002)$ como de $P$. halepensis $(\mathrm{r}=-0,3415 ; P<0,0001)$. Sin embargo, no se observaron correlaciones significativas entre los valores de $\mathrm{AB}_{\text {local }} \mathrm{y}$ de temperatura del suelo para ninguna de las dos especies.

\section{DISCUSIÓN}

El desfronde medio anual observado en los rodales de $P$. sylvestris es ligeramente inferior al observado por Santa-Regina y Tarazona (2001) en una repoblación de P. sylvestris en la Sierra de la Demanda (España), que fue de $2.907 \mathrm{~kg} \mathrm{ha}^{-1}$ y por Gallardo-Lancho y Santa-Regina (1991) en la Sierra de Béjar, que fue de $3.631 \mathrm{~kg} \mathrm{ha}^{-1}$. En el presente trabajo se ha observado que el pico de mayor desfronde en P. sylvestris se produce en los meses de julio a septiembre siendo agosto el mes de mayor desfronde. Estos resultados difieren ligeramente de los encontrados por autores como Pausas (1997) o Blanco et al. (2005) en masas de $P$. sylvestris de Pirineos (España), los cuales observaron el pico de mayor desfronde en los meses de agosto a

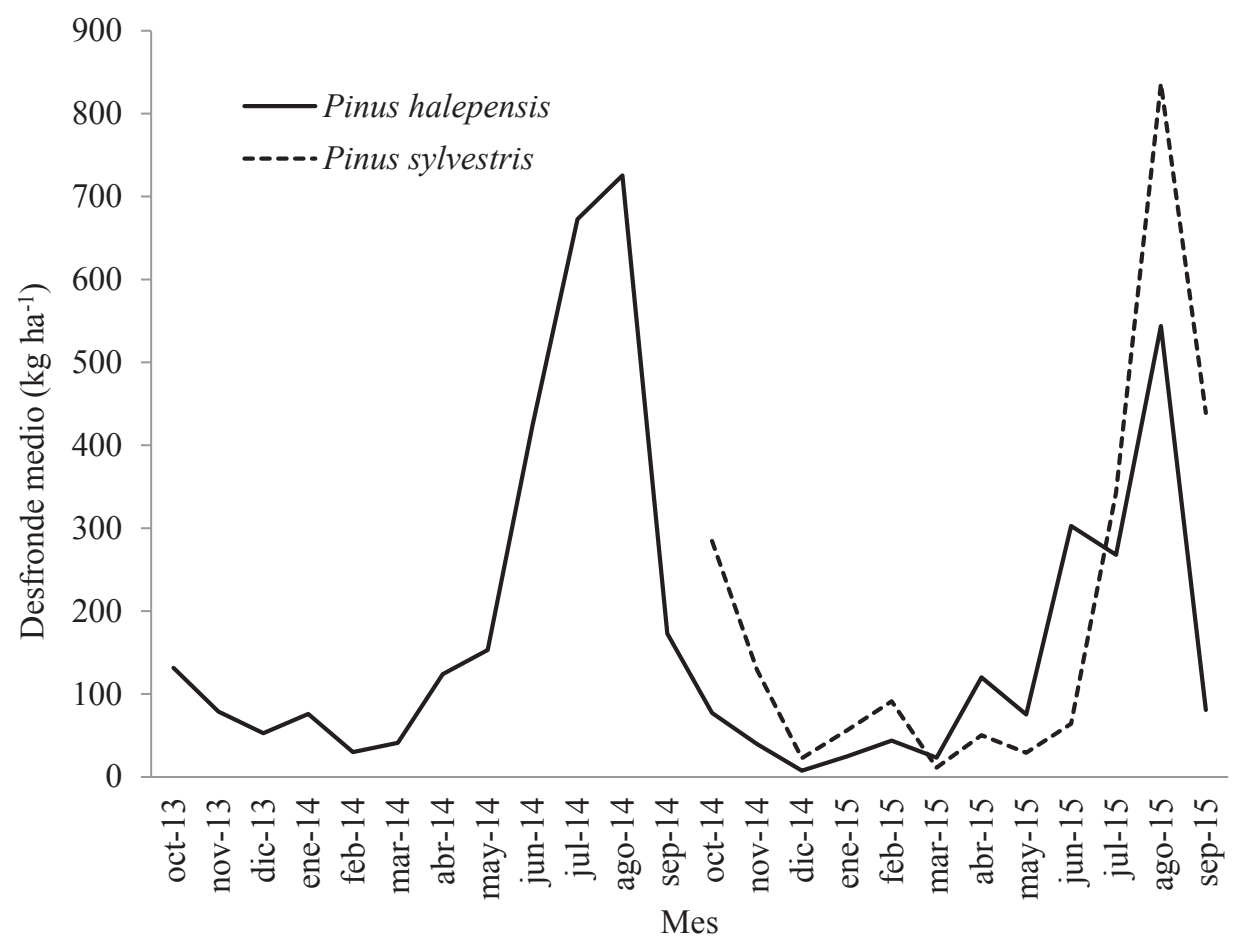

Figura 2. Producción media mensual de desfronde de acículas observada en los rodales de Pinus sylvestris y Pinus halepensis estudiados.

Mean monthly needle-fall productions in the studied Pinus sylvestris and Pinus halepensis stands. 
A)

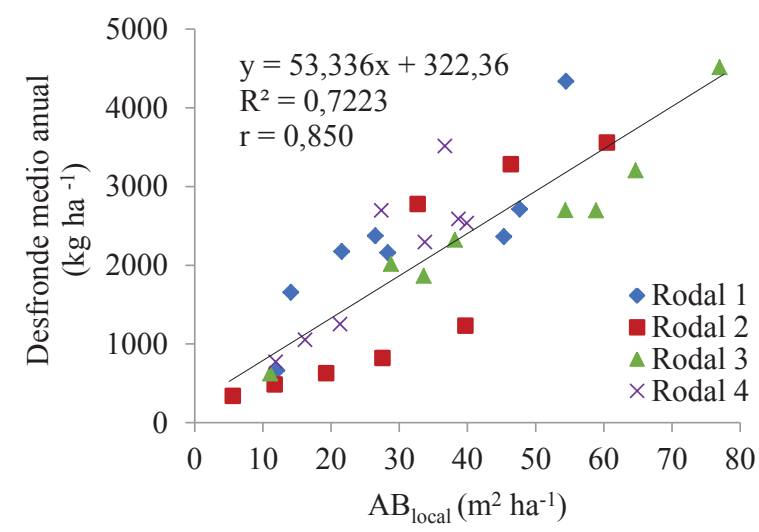

B)

Pinus sylvestris

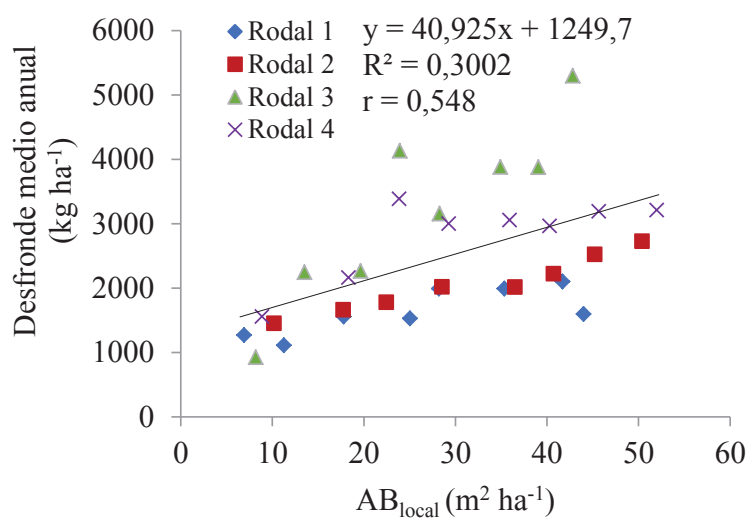

Figura 3. Relación entre el desfronde medio anual recogido y el $\mathrm{AB}_{\text {local }}$ de las parcelas estudiadas: A) Pinus halepensis, B) Pinus sylvestris.

Relationship between mean annual needle litterfall production and local basal area in the plots studied.

Cuadro 2. Estimadores de la tasa de descomposición $\left(\mathrm{k} ;\right.$ meses $\left.^{-1}\right)$, tiempo de vida medio de las acículas $\left(\mathrm{t}_{0,5} ;\right.$ meses), tasas lineales de cambio de los mismos $(\beta)$ por unidad de área basimétrica y valores $P$ según el análisis de la varianza del modelo lineal mixto ajustado.

Decay rate coefficients $(\mathrm{k})$, half-life for the decomposing litter $\left(\mathrm{t}_{0,5}\right)$, linear change rate $(\beta)$ per unit of basal area and $P$ values given by the analysis of variance of the linear mixed model.

\begin{tabular}{ccccc}
\hline Especie & Parámetro & Estimador & $\beta$ & $P$ \\
\hline \multirow{2}{*}{ Pinus halepensis } & $\mathrm{k}$ & 0,0248 & $-0,00009$ & $<0,0001$ \\
& $\mathrm{t}_{0,5}$ & 29,3 & 0,12530 & $<0,0001$ \\
\hline \multirow{2}{*}{ Pinus sylvestris } & $\mathrm{k}$ & 0,0306 & $-0,00002$ & $\mathrm{~ns}$ \\
& $\mathrm{t}_{0,5}$ & 22,8 & 0,01616 & $\mathrm{~ns}$ \\
\hline
\end{tabular}

A)

Pinus halepensis

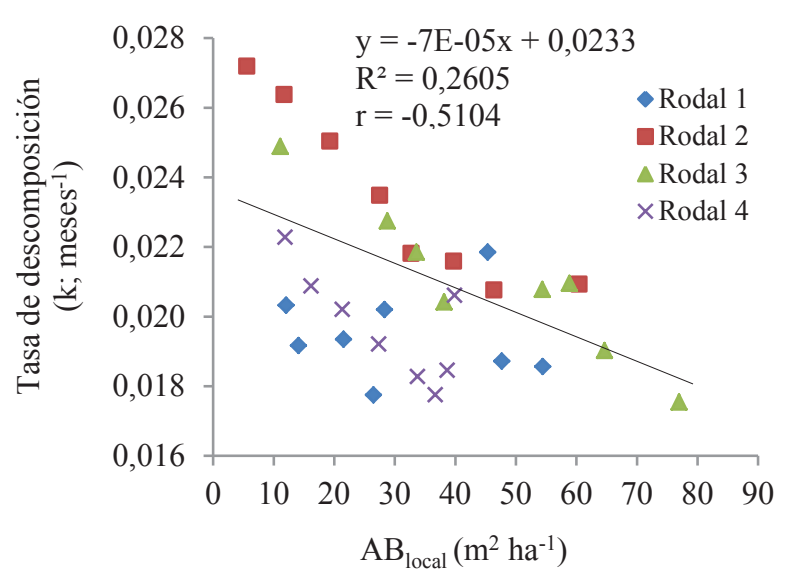

B)

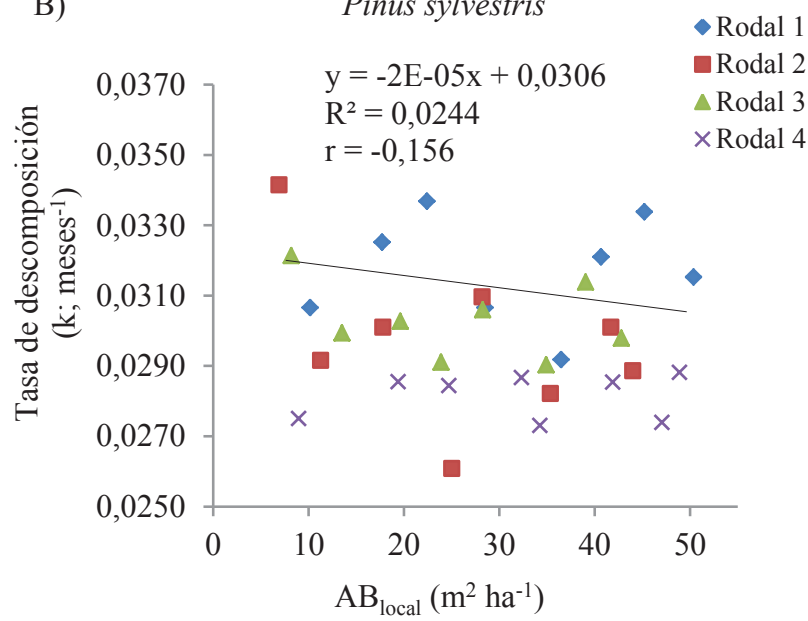

Figura 4. Relación entre la tasa de descomposición observada en las bolsas de descomposición y el $\mathrm{AB}_{\text {local }}$ de las parcelas estudiadas: A) Pinus halepensis, B) Pinus sylvestris.

Relationship between the decay rates and local basal area in the plots studied. 
octubre. Las tasas de desfronde observadas en los rodales de $P$. halepensis en el presente trabajo son similares a las encontradas por Navarro et al. (2013) en el sur de España (entre $950 \mathrm{~kg} \mathrm{ha}^{-1}$ y $2.280 \mathrm{~kg} \mathrm{ha}^{-1}$ ), quienes observaron que los meses de mayor desfronde eran desde julio hasta octubre. En las parcelas de P. halepensis del presente estudio los meses de mayor desfronde son junio, julio y agosto. Los pequeños desfases observados en relación a los meses de máximo desfronde entre los distintos trabajos pueden deberse a la fenología de las especies y a las diferencias climáticas existentes entre las zonas de los distintos estudios ya que, como describieron Escudero y del Arco (1987), el momento en que se produce la abscisión foliar viene determinado por el estrés hídrico, directamente relacionado con las condiciones climáticas (Roig et al. 2005). Se trata de una adaptación de las especies forestales frente al déficit hídrico mediante la cual se disminuye la superficie de transpiración disminuyendo así las pérdidas de agua.

El efecto del $\mathrm{AB}_{\text {local }}$ sobre la producción de desfronde observado en las masas de ambas especies ha sido significativo y positivo y coincide con lo descrito previamente por diversos autores para distintas especies (Kunhamu et al. 2009, Navarro et al. 2013, Lado-Monserrat et al. 2015), lo cual indica que una mayor espesura (medida en términos de $\mathrm{AB}_{\text {local }}$ ) conlleva una mayor biomasa de acículas por unidad de superficie en la masa forestal y, por tanto, una mayor cantidad de desfronde.

En cuanto a la descomposición de acículas en $P$. sylvestris no se han hallado diferencias significativas durante el primer año en función del $\mathrm{AB}_{\text {local }}$ de la parcela. Por otra parte, en las masas de $P$. halepensis sí se ha observado un efecto significativo del $\mathrm{AB}_{\text {local }}$ sobre la velocidad de descomposición de las acículas $\left(\mathrm{k} \mathrm{y}_{0,5}\right)$ en los dos años estudiados. Lado-Monserrat et al. (2015) observaron una disminución significativa de la tasa de descomposición de la hojarasca en las zonas sujetas a cortas a hecho respecto al tratamiento control (masa no aclarada) en masas de $P$. halepensis del este de España. Sin embargo, no hallaron diferencias significativas entre el tratamiento control y distintos niveles de clara. Asimismo, en dicho trabajo el material extraído de las bolsas presentaba mayor humedad en las parcelas control en los periodos secos. En el presente estudio se ha observado una tendencia contraria, es decir, una tasa de descomposición mayor en las parcelas con menor $\mathrm{AB}_{\text {local }}$ y una correlación significativa y negativa entre el $\mathrm{AB}_{\text {local }}$ y la humedad del suelo de manera que las zonas con menor $\mathrm{AB}_{\text {local }}$ se ha observado mayor humedad. Desanto et al. (1993) observaron también una relación entre la pérdida de peso de las acículas y un parámetro relacionado con la humedad del suelo, en este caso, la precipitación diaria en masas de P. sylvestris. Por tanto, parece que la disponibilidad de humedad es un factor decisivo para la descomposición pero la densidad de la masa no provoca los mismos efectos sobre el microclima a nivel de suelo en las distintas zonas climáticas. Parece evidente que el proceso que prevalece en la zona de estudio es la interceptación de las precipitaciones por parte de las copas de los árboles, desde las cuales la humedad se evapora no llegando al suelo en las zonas de mayor densidad. En otros trabajos, el proceso predominante parece ser el incremento de la radiación que alcanza el suelo en las zonas con menos cobertura, incrementando por tanto la temperatura del suelo y disminuyendo la humedad (Lado-Monserrat et al. 2015). Por tanto, en las masas de P. halepensis estudiadas se observa una mayor velocidad de descomposición de las acículas en las parcelas con menor $\mathrm{AB}_{\text {local }}$ donde a su vez se ha observado una mayor humedad, lo cual posibilita una mayor actividad de los microorganismos responsables de la descomposición de la materia orgánica. Sin embargo, en las masas de P. sylvestris estudiadas dicho efecto no ha resultado significativo, posiblemente por el mayor régimen de precipitaciones existente, donde la humedad parece no ser un factor limitante para los microorganismos descomponedores, pudiendo producirse así un desempeño similar de los microorganismos descomponedores a distintos niveles de $\mathrm{AB}_{\text {local }}$.

\section{CONCLUSIONES}

Las tasas de desfronde observadas en el presente trabajo difieren ligeramente de las obtenidas por otros autores para estas mismas especies en otras zonas de España, observándose un pequeño desfase en relación a los meses en los que se observan las mayores tasas de desfronde. Asimismo, el área basimétrica de las masas tiene una influencia significativa y positiva sobre el desfronde y negativa sobre la descomposición de la hojarasca, debido este último efecto principalmente a la disminución de la humedad en la superficie del suelo. Estos efectos deben ser tenidos en cuenta en la planificación de las prácticas selvícolas ya que determinan en buena medida la disponibilidad de nutrientes edáficos y por tanto, el estado nutricional de las masas.

\section{AGRADECIMIENTOS}

Los autores agradecen a Elisa Mellado y Olga López su apoyo en el trabajo de campo y a Carmen Blanco y Juan Carlos Arranz su consejo en el trabajo de laboratorio. Este trabajo se ha financiado gracias a los proyectos AGL201129701-C02-02 y AGL2014-51964-C2-1-R del Ministerio de Economía y Competitividad del Gobierno de España y a la beca predoctoral concedida a T. Bueis por la Universidad de Valladolid y el Banco Santander.

\section{REFERENCIAS}

Aceñolaza PG, JF Gallardo-Lancho. 1995. Influencia de la edad del bosque en la evolución de la pérdida de peso seco en hojarasca de Alnus acuminata en alisedas de la provincia de Tucumán (Argentina). Investigación agraria. Sistemas y Recursos Forestales 4(2): 177-188. 
Blanco JA, MA Zavala, JB Imbert, FJ Castillo. 2005. Sustainability of forest management practices: Evaluation through a simulation model of nutrient cycling. Forest Ecology and Management 213(1-3): 209-228. DOI: 10.1016/j.foreco.2005.03.042.

Desanto AV, B Berg, FA Rutigliano, A Alfani, A Fioretto. 1993. Factors regulating early-stage decomposition of needle litters in 5 different coniferous forests. Soil Biology \& Biochemistry 25(10): 1423-1433. DOI: 10.1016/00380717(93)90057-i.

Escudero A, JM del Arco. 1987. Ecological significance of the phenology of leaf abscission. Oikos 49: 11-14. DOI: $10.2307 / 3565549$.

Gallardo-Lancho JF, I Santa-Regina, C San Miguel. 1989. Ciclos biogeoquímicos en bosques de la Sierra de Béjar: 1. Producción de la hojarasca. Revue d'Ecologie et de Biologie du Sol 26(1): 35-46.

Kunhamu TK, BM Kumar, S Viswanath. 2009. Does thinning affect litterfall, litter decomposition, and associated nutrient release in Acacia mangium stands of Kerala in peninsular India? Canadian Journal of Forest Research-Revue Canadienne De Recherche Forestiere 39(4): 792-801. DOI: 10.1139/x09-008.

Lado-Monserrat L, A Lidon, I Bautista. 2015. Litterfall, litter decomposition and associated nutrient fluxes in Pinus halepensis: influence of tree removal intensity in a Mediterra- nean forest. European Journal of Forest Research 134(5): 833-844. DOI: 10.1007/s10342-015-0893-z.

Martínez-Alonso C, F Valladares, JJ Camarero, M López Arias, M Serrano, JA Rodríguez. 2007. The uncoupling of secondary growth, cone and litter production by intradecadal climatic variability in a Mediterranean Scots pine forest. Forest Ecology and Management 253(1): 19-29.

Navarro FB, A Romero-Freire, T Del Castillo, A Foronda, MN Jimenez, MA Ripoll, A Sanchez-Miranda, L Huntsinger, E Fernandez-Ondono. 2013. Effects of thinning on litterfall were found after years in a Pinus halepensis afforestation area at tree and stand levels. Forest Ecology and Management 289: 354-362. DOI: 10.1016/j.foreco.2012.09.026.

Olson JS. 1963. Energy storage and balance of producers and decomposers in ecological systems. Ecology 44(2): 322-331. DOI: $10.2307 / 1932179$.

Pausas JG. 1997. Litter fall and litter decomposition in Pinus sylvestris forests of the eastern Pyrenees. Journal of Vegetation Science 8(5): 643-650. DOI: 10.2307/3237368.

Roig S, M del Rio, I Canellas, G Montero. 2005. Litter fall in Mediterranean Pinus pinaster Ait. stands under different thinning regimes. Forest Ecology and Management 206(13): 179-190. DOI: 10.1016/j.foreco.2004.10.068.

Santa-Regina I, T Tarazona. 2001. Nutrient cycling in a natural beech forest and adjacent planted pine in northern Spain. Forestry 74(1): 11-28. DOI: 10.1093/forestry/74.1.11. 
